\title{
Imaging protective mast cells in living mice during severe contact hypersensitivity
}

\author{
Laurent L. Reber, ${ }^{1,2}$ Riccardo Sibilano, ${ }^{2,3}$ Philipp Starkl, ${ }^{2,4}$ Axel Roers, ${ }^{5}$ Michele A. Grimbaldeston, ${ }^{6}$ \\ Mindy Tsai, ${ }^{2,3}$ Nicolas Gaudenzio, ${ }^{2,3}$ and Stephen J. Galli2,3,7 \\ 'Department of Immunology, Unit of Antibodies in Therapy and Pathology, Institut Pasteur, Paris, France; INSERM, U1222, \\ Paris, France. ${ }^{2}$ Department of Pathology, ${ }^{3}$ Sean N. Parker Center for Allergy and Asthma Research, Stanford University \\ School of Medicine, California, USA. ${ }^{4}$ CeMM Research Center for Molecular Medicine of the Austrian Academy of Sciences, \\ and Department of Medicine I, Medical University of Vienna, Vienna, Austria. Institute for Immunology, University of \\ Technology Dresden, Medical Faculty Carl-Gustav Carus, Dresden, Germany. ${ }^{6} \mathrm{OMNI}$-Biomarker Development, Genentech \\ Inc., South San Francisco, California, USA. 'Department of Microbiology \& Immunology Stanford University School of \\ Medicine, Stanford, California, USA.
}

Contact hypersensitivity (CHS) is a common skin disease induced by epicutaneous sensitization to haptens. Conflicting results have been obtained regarding pathogenic versus protective roles of mast cells (MCs) in CHS, and this has been attributed in part to the limitations of certain models for studying MC functions in vivo. Here we describe a fluorescent imaging approach that enables in vivo selective labeling and tracking of MC secretory granules by real-time intravital 2-photon microscopy in living mice, and permits the identification of such MCs as a potential source of cytokines in different disease models. We show using this method that dermal MCs release their granules progressively into the surrounding microenvironment, but also represent an initial source of the antiinflammatory cytokine IL-10, during the early phase of severe CHS reactions. Finally, using 3 different types of MC-deficient mice, as well as mice in which IL-10 is ablated specifically in MCs, we show that IL-10 production by MCs can significantly limit the inflammation and tissue pathology observed in severe CHS reactions.

Conflict of interest: The authors have declared that no conflict of interest exists.

Authorship note: R. Sibilano and P. Starkl contributed equally to this work. N. Gaudenzio and S.J. Galli contributed equally to this work.

Submitted: January 18, 2017 Accepted: March 23, 2017 Published: May 4, 2017

Reference information: JCI Insight. 2017;2(9):e92900. https:// doi.org/10.1172/jici.nsight.92900.

\section{Introduction}

One important function of the immune system is to restrain the tissue pathology that can occur during severe inflammatory reactions. Mast cells (MCs) are widely deployed at host-environment interfaces (e.g., the skin or mucosae) and can be activated to secrete diverse products, including some with antiinflammatory or immunosuppressive functions, in response to a variety of different stimuli (1-5). MCs therefore can represent both powerful sentinels of the immune system and potential sources of products which might limit inflammation or its associated tissue injury.

However, whether MCs actually perform antiinflammatory or immunosuppressive functions in vivo is controversial. We proposed, based on experiments performed in mice with a profound MC deficiency related to abnormalities in Kit structure or expression (referred to herein as "Kit-mutant MC-deficient mice"), that MCs and MC production of IL-10 can limit skin pathology during severe contact hypersensitivity (CHS) reactions (6). As noted in that study, it has been appreciated for some time that Kit-mutant mice have several phenotypic abnormalities in addition to their MC deficiency, and subsequently we and others generated Kit-independent MC-deficient mouse strains that are deficient in MC populations due to genetic manipulations independent of alterations in Kit (7-12).

Using one Kit-independent MC-deficient strain, Dudeck et al. reported evidence that MCs amplify rather than limit CHS responses, and concluded that the exaggerated CHS reactions observed in Kit-mutant animals are not caused by the absence of MCs but instead reflect other effects of the KIT deficiency $(7,13)$. By contrast, based on findings in both Kit-mutant and Kit-independent MC-deficient mice, Gimenez-Rivera et al. recently reported that MCs can suppress skin inflammation in a new mouse model of chronic CHS (14).

To help resolve the apparent controversy about whether MCs and MC-derived IL-10 indeed can function to suppress severe acute CHS, we developed a new imaging method that permits the unambiguous 
monitoring, in dermal MCs in vivo, of both changes in their cytoplasmic secretory granules and activation of $\mathrm{Il10}$ gene transcription. In contrast to approaches employing genetically encoded cell tracers, which usually require breeding of the reporter mice with other strains to achieve cell-specific expression of the tracer, our new tunable fluorescent imaging approach can (a) be employed regardless of mouse strain, (b) enable in vivo selective labeling of MC secretory granules for prolonged time periods, and (c) permit longitudinal monitoring of such labeled MCs in vivo by noninvasive intravital 2-photon microcopy.

Using this new in vivo imaging method, we monitored simultaneously both MC granule secretion dynamics and MC activation of transcription of the $I 110$ gene in our model of 1-fluoro-2,4-dinitrobenzeneinduced (DNFB-induced) severe CHS. We show that dermal MCs secrete intracellular granule content into the surrounding microenvironment a few hours after epicutaneous DNFB challenge, and that MCs also represent one of the first immune cells to exhibit $I l 10$ gene activation at such sites. Finally, using 2 different strains of Kit-independent MC-deficient mice, as well as mice in which IL-10 is specifically ablated in MCs, we confirmed that MCs, and MC-derived IL-10, can significantly limit both the inflammation and the tissue pathology observed in severe CHS reactions.

\section{Results}

In vivo labeling and imaging of dermal MC cytoplasmic granules. We recently reported a method to visualize the spatiotemporal features of MC granule structures once they have been exteriorized during MC degranulation in vivo: intradermal (i.d.) injection of fluorochrome-labeled avidin (sulforhodamine 101-coupled avidin [Av.SRho]) into the ear pinna dermis 10 to 30 minutes before performing 2-photon microscopy (15). During MC degranulation, which results in fusion of secretory granule membranes with the plasma membrane $(16,17)$, externalized granule matrices are rapidly bound by soluble fluorescent Av.SRho probe present in the microenvironment, allowing imaging by high-resolution confocal and in vivo 2-photon microscopy of such exteriorized granule structures $(15,18)$. While this method is useful for identifying exteriorized MC granule structures, it requires (a) that MCs are activated to degranulate, and (b) using specific MC reporter mice (e.g., Mcpt5-EYFP mice; see refs. 7, 19) to identify nondegranulated MCs in the dermis prior to their activation (therefore limiting the use of other reporter animal models encoding fluorescent tracers of comparable excitation/emission spectra).

To bypass these limitations, we investigated whether long-term exposure (i.e., days to months) to fluorochrome-labeled avidin in vivo would result in uptake of the probe and specific labeling of intracellular secretory granules of dermal MCs under homeostatic conditions. Seven days after performing single i.d. injections of Av.SRho into the ear pinna dermis of $\mathrm{Kit}^{+/+}$(MC-sufficient) or $\mathrm{Kit}^{\mathrm{W}-\mathrm{s} h / W-\mathrm{sh}}$ (MC-deficient) mice, we examined the living mice by 2-photon microscopy to characterize the size, shape, and intensity of Av.SRho fluorescence signals, and their location with respect to blood vessels (identified after intravenous [i.v.] injection of FITC-labeled dextran; see refs. 15, 20) and hair follicle areas (corresponding to regions of low collagen content and identified by a second harmonic generation signal; see ref. 21) (Figure 1A). We identified many Av.SRho+ fluorescent granulated cells that morphologically resembled MCs in the dermis of MC-sufficient $\mathrm{Kit}^{+/+}$mice, often located near blood vessels and hair follicles. By contrast, such fluorescent cells were completely absent (i.e., no detection of red fluorescence above the red autofluorescence of the dermis) in MC-deficient $K i t^{W-s h / W-s h}$ mice (Figure 1, B-D). Ex vivo analyses of Av.SRho-injected sites in $\mathrm{Kit}^{+/+}$mice showed that the Av.SRho ${ }^{+}$granular cells also expressed the stem cell factor receptor KIT (Supplemental Figure 1A; supplemental material available online with this article; https://doi.org/10.1172/jci. insight.92900DS1), which is highly expressed at the MC surface (22).

To confirm unambiguously that the observed Av.SRho ${ }^{+}$granular cells were indeed $\mathrm{MCs}$, we infused Av.SRho into the ear pinna of Mcpt5-EYFP mice (Mcpt5-EYFP mice (in which Cre expression driven by the mast cell protease 5 [Mcpt5] promoter restricts expression of the Cre-inducible EYFP transgene to connective tissue-type MCs; see refs. 7, 19) (Figure 1E), and analyzed the spatial overlap of Av.SRho and EYFP fluorescence signals employing high-resolution 3D single-cell 2-photon imaging, as previously described (15). We observed an almost complete spatial overlap of the 2 fluorescent signals (Figure 1F). When fluorescence overlap was quantified at the single-cell level in the Av.SRho-injected area, we confirmed that $\sim 90 \%$ of EYFP E $^{+}$ dermal MCs were also Av.SRho ${ }^{+}$, while we detected hardly any single-positive $\left(\mathrm{EYFP}^{-} \mathrm{Av} . \mathrm{SRho}^{+}\right.$or $\mathrm{EYFP}^{+} \mathrm{Av}$. $\mathrm{SRho}^{-}$) cells (Figure 1, G and H). Finally, we detected Av.SRho ${ }^{+} \mathrm{MCs}$ as early as 3 days following i.d. injection of the probe, and such fluorescent signal stayed bright and stable for at least 120 days after injection in resting mice (no later time points were investigated) (Supplemental Figure 1B). This long-term storage of the dye in 
A

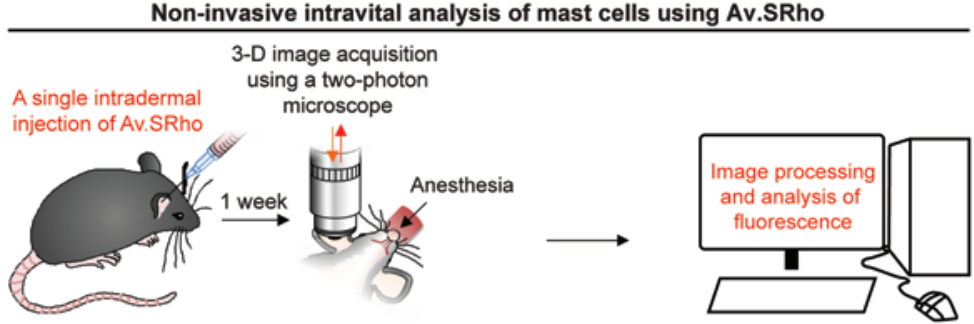

B

70 kd Dextran-FITC Av.SRho Collagen
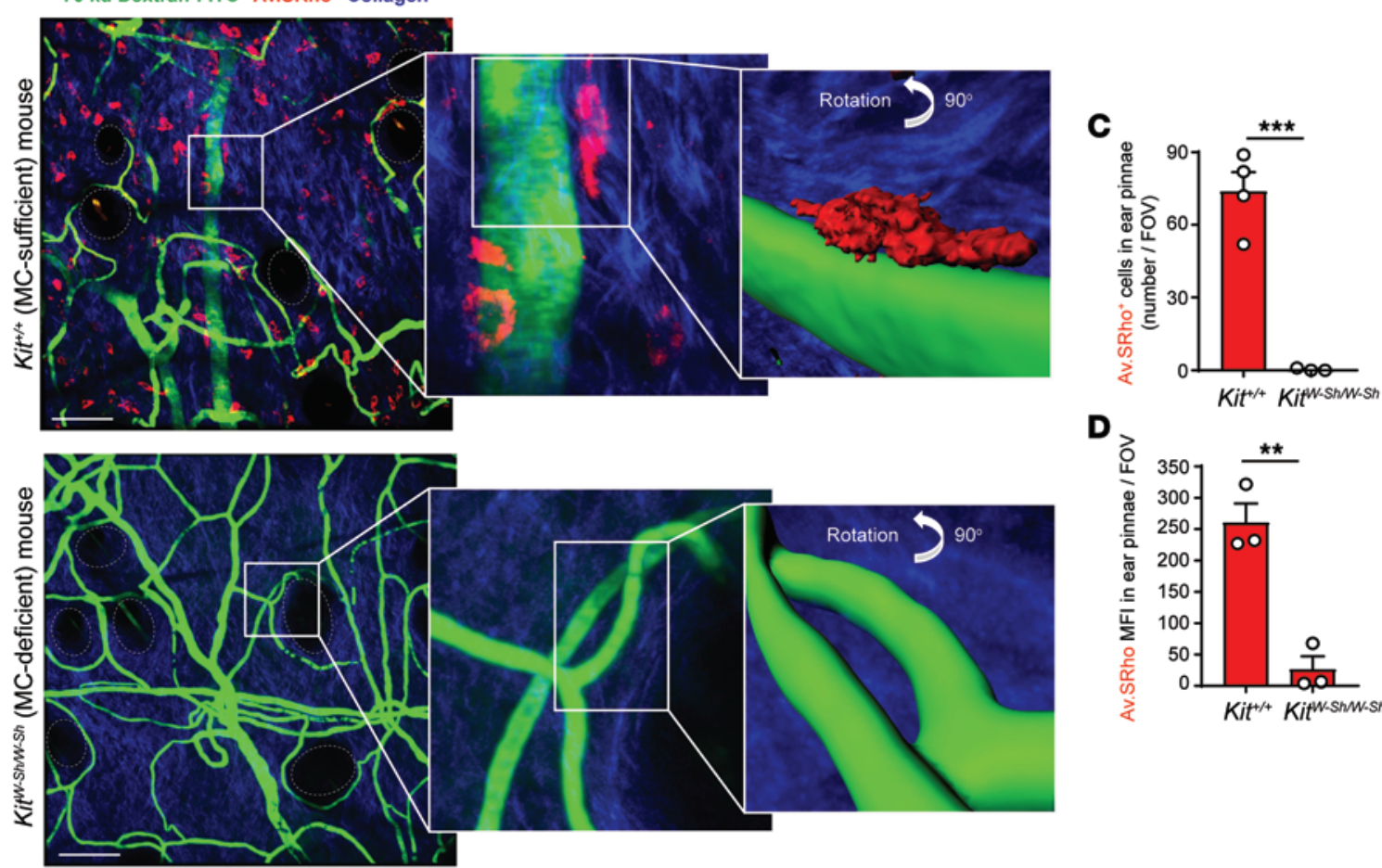

E

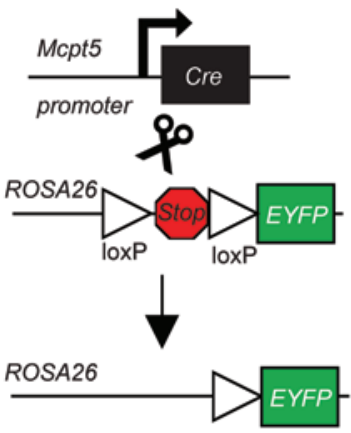

$\mathbf{F}$

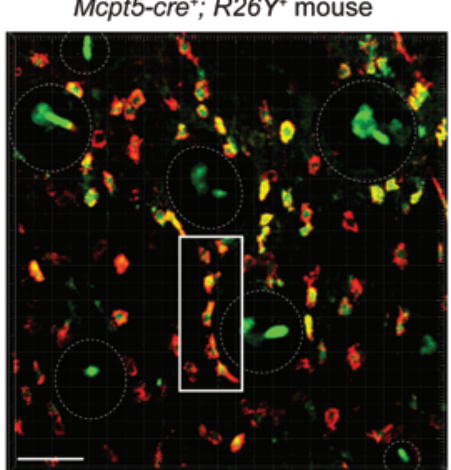

Av.SRho

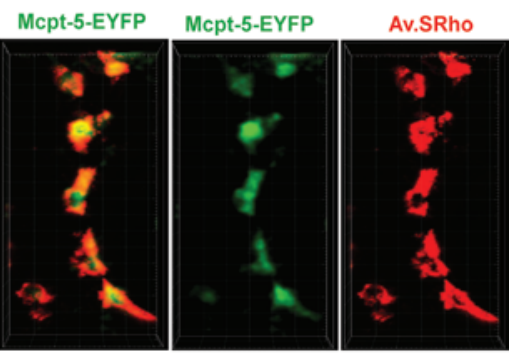

Skin and peritoneal mast cells
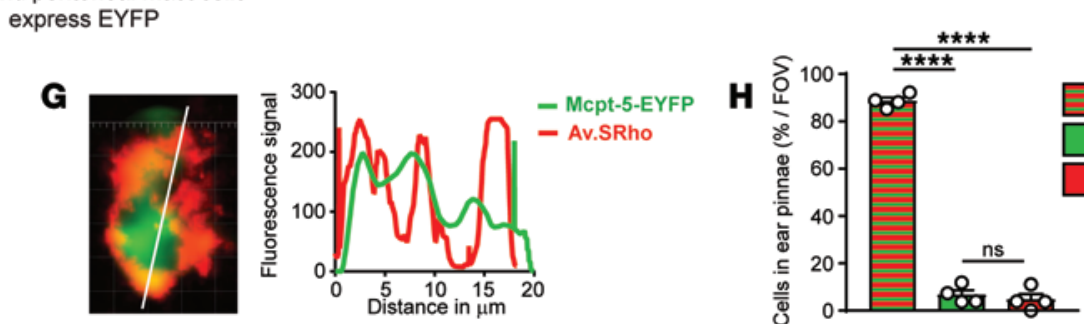

Mcpt-5-EYFP* Av.SRho

Mcpt-5-EYFP*

Av.SRho ${ }^{+}$

Figure 1. A single injection of fluorochrome-labeled avidin enables specific labeling of dermal mast cell (MC) intracellular secretory granules. (A) Protocol: $5 \mu$ g of sulforhodamine 101-coupled avidin (Av.SRho) was injected intradermally (i.d.) into the ear pinna of a mouse. One week later the mouse was anesthetized and placed under a 2-photon microscope; 3D high-resolution images were taken and Av.SRho fluorescence signal was assessed. (B) $\mathrm{Kit}^{+/+}$MC-sufficient (upper panel) or Kit ${ }^{W-s h / W-s h}$ MC-deficient (lower panel) mice were treated as described in $\mathbf{A}$ except that $250 \mu \mathrm{g}$ of $70-\mathrm{kDa}$ dextran-FITC 
was injected retro-orbitally 30 minutes before imaging to label blood vessels. Representative 3D photographs of the ear pinna showing merged fluorescence of Av.SRho (red), dextran-FITC (green), and collagen structures (blue). White lines identify the magnified areas. Far right panel: Av.SRho (red) and dextran-FITC (green) fluorescence was modeled and the image was rotated $90^{\circ}$ to better depict the modeled fluorescence signals. (C) Number of Av.SRho ${ }^{+}$ cells in ear pinnae per field of view (FOV) in $\mathrm{Kit}^{+/+}$or $\mathrm{Kit}^{\mathrm{W} \text {-sh/W-sh }}$ mice. (D) Av.SRho mean fluorescence intensity (MFI) per FOV in $\mathrm{Kit}^{+/+}$or $\mathrm{Kit}^{\mathrm{W} \text {-sh/W-sh }}$ mice. (E) Breeding strategy to obtain Mcpt5-Cre+; R26Y+ (Mcpt5-EYFP) mice. (F) Mcpt5-EYFP mice were treated as described in A. Representative 3D photographs of the ear pinna showing merged fluorescence of Av.SRho (red) and EYFP (green). White lines identify the magnified area, dashed white circles identify hair follicles. (C) Left panel: 3D photographs of a single Av.SRho+ EYFP+ cell. Right panel: spatial localization of the Av.SRho (red) and EYFP (green) fluorescence along the white line depicted in the left panel. (H) Percentage of Av.SRho ${ }^{+}$EYFP $^{+}$(red and green), Av.SRho-EYFP+ (green) and Av.SRho+EYFP- (red) cells in ear pinnae per field of view (FOV) in Mcpt5-EYFP mice. Mean + SEM, 2-tailed, unpaired $t$ test, ${ }^{* *} P<0.01 ;{ }^{* *} P<0.001$; ${ }^{* * * *} P<0.0001$. Data $(n=3$ mice per group) are pooled from the 3 experiments performed (each done with 1 mouse per group), each of which gave similar results; circles show values for individual mice. Scale bars: $100 \mu \mathrm{m}$.

MC granules is likely due to the presence in the granules of high levels of negatively charged proteoglycans (e.g., heparin), to which avidin can strongly bind $(18,23,24)$, as well as to the low $\mathrm{pH}$ of $\mathrm{MC}$ granules, which might protect the avidin probe from protease-mediated degradation (25). Taken together, these results demonstrate that a single i.d. injection of fluorochrome-labeled avidin results in highly selective labeling of the cytoplasmic granules of dermal MCs, thus enabling their visualization by noninvasive intravital microscopy in living mice for a prolonged period of time.

Granule-associated avidin does not interfere with MC degranulation or MC-dependent PCA reactions. Avidin molecules have a strong affinity for highly negatively charged molecules in MC secretory granules, e.g., heparin (26). We therefore investigated whether incorporation of fluorochrome-labeled avidin could interfere with MC degranulation, the release of granule-associated mediators, or the induction of classical MC-dependent allergic inflammation at sites of IgE-dependent passive cutaneous anaphylaxis (PCA). We incubated in vitro bone marrow-derived cultured MCs (BMCMCs) with Av.SRho for 4 days (the time required to observe substantial incorporation of Av.SRho into their secretory granules [Supplemental Figure $1 \mathrm{C}]$ ) and measured the quantity of $\beta$-hexosaminidase release (27) in their supernatants upon $\mathrm{IgE} / \mathrm{Ag}$ challenge. While we barely detected the presence of $\beta$-hexosaminidase in supernatants of nonstimulated control BMCMCs, we measured comparable quantities of the enzyme in the supernatants of Av.SRho- or Av.SRho ${ }^{+}$BMCMCs stimulated with IgE/Ag (Supplemental Figure 1D).

We also examined IgE-dependent PCA reactions (28) to assess whether the incorporation of Av.SRho into the cytoplasmic granules of dermal MCs might influence either antigen-induced MC degranulation or 2 of the MC-dependent features of such reactions: (a) local tissue swelling, or (b) infiltration of immune cells into the ear pinna. We used noninvasive 2-photon microscopy to monitor simultaneously the release of MC cytoplasmic granules and the associated changes in local blood flow (by measuring changes in fluorescence intensity in the interstitial space in mice injected i.v. with dextran-FITC; see refs. 15, 20) upon induction of IgE-mediated PCA. In mice injected with vehicle, little or no MC activation was observed and minimal amounts of dextran-FITC extravasation were detected in the interstitial space (Figure 2A and Supplemental Video 1). In agreement with our recent report describing the dynamics of MC responses upon their activation by different stimuli (15), induction of an IgE-mediated PCA reaction was associated with the delayed (starting $\sim 15$ minutes after challenge) but progressive release of Av.SRho ${ }^{+}$cytoplasmic secretory granules, some of which then appeared to be transported further within the tissue matrix (Figure 2A and Supplemental Video 2). In accord with this observation, we also measured a relatively slow (also starting $\sim 15$ minutes after challenge) but progressive extravasation of dextran-FITC, with the highest levels detected in the interstitial space $\sim 25$ minutes after induction of the reaction (Figure 2, A and B, and Supplemental Video 2). Interestingly, we noticed that the extravasation of dextran-FITC always started prior to the detection of exteriorized Av.SRho ${ }^{+}$granule structures (Figure 2B). These data are consistent with current understanding that histamine, which can augment vascular permeability, is one of the first cytoplasmic granule-associated mediators released upon MC activation.

We next measured the swelling elicited during reactions induced in both the vehicle-infused (Av.SRho-, left) or contralateral Av.SRho-injected (Av.SRho ${ }^{+}$, right) IgE-sensitized ear pinnae in each animal (each animal serves as its own control), upon i.p. injections of vehicle (control) or 2,4-dinitrophenyl-conjugated human serum albumin (DNP-HSA, to induce a PCA reaction). I.p. injection of vehicle induced a weak and transient tissue swelling response in some mice that resolved within $\sim 60$ to 90 minutes (Figure 2C). Challenge with DNP-HSA induced strong ear swelling, with the highest levels at 30 minutes after challenge and requiring $\sim 6$ hours to resolve fully (Figure $2 \mathrm{C}$ ). We detected similar levels of ear swelling at all time points 
A 70 kd Dextran-FITC Av.SRho Collagen
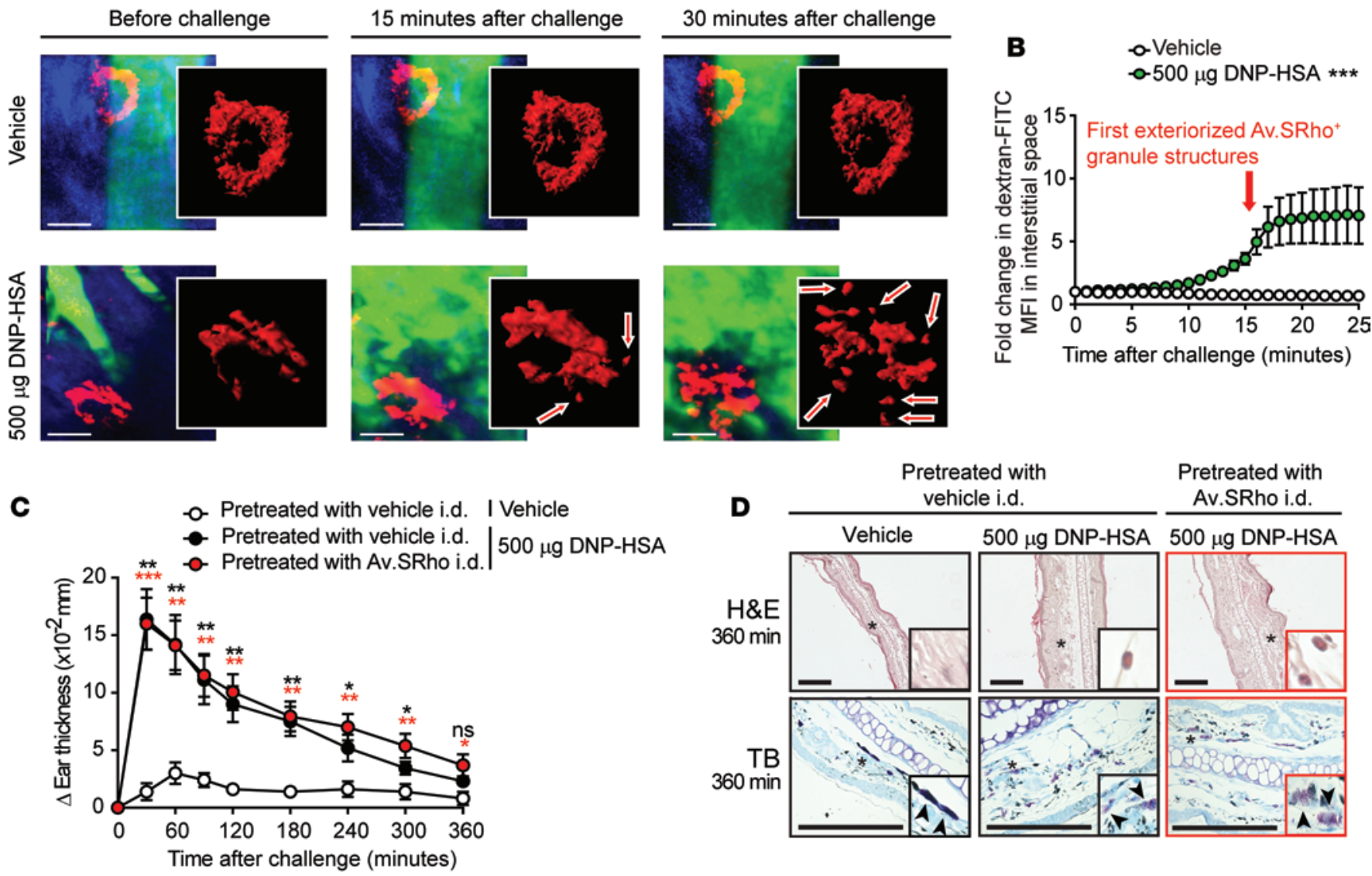

$\mathbf{E}$

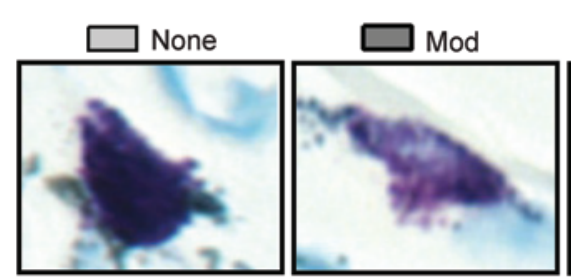

Ext
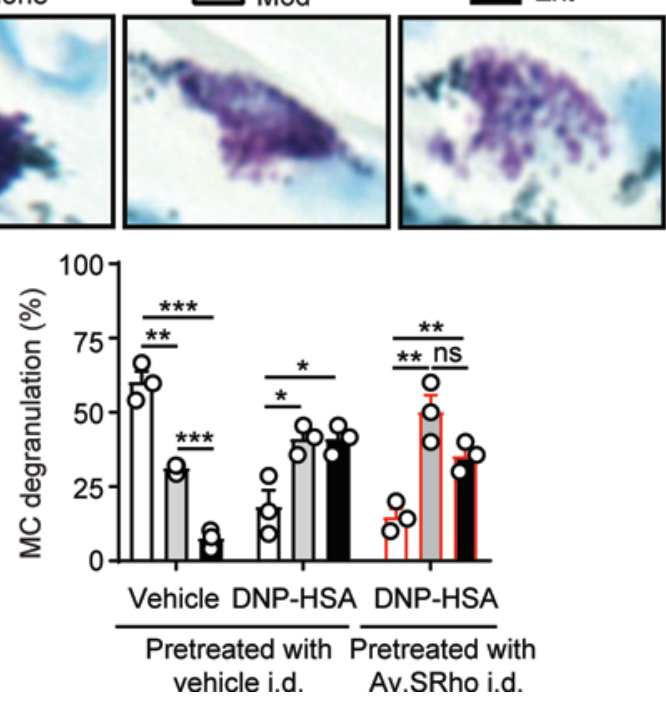

$\mathbf{F}$
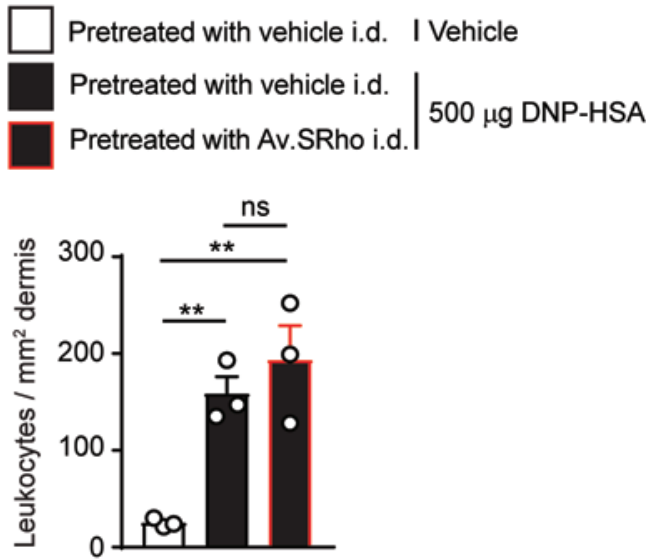

Figure 2. Intradermal injection of fluorochrome-labeled avidin does not interfere with IgE-mediated mast cell (MC) degranulation or IgE- and MCdependent inflammation in vivo. (A and B) Sulforhodamine 101-coupled avidin (Av.SRho; $5 \mu \mathrm{g}$ ) was injected intradermally (i.d.) into the ear pinna of C57BL/ 6 mice. One week later mice were sensitized by i.d. injection of $20 \mathrm{ng}$ of mouse anti-2,4-dinitrophenyl (anti-DNP) IgE into the ear pinna. Sixteen hours later, $250 \mu \mathrm{g}$ of 70-kDa dextran-FITC was injected retro-orbitally and the anesthetized mice were positioned under the 2-photon microscope. AntiDNP IgE-sensitized mice were injected i.p. with $500 \mu \mathrm{g}$ of DNP-conjugated human serum antigen (DNP-HSA) or with vehicle (control). Image sequences were acquired in $3 \mathrm{D}$ at a rate of 1 picture per minute over 30 minutes using a 2-photon microscope. (A) Representative 3D time-lapse sequences of merged Av.SRho (red), dextran-FITC (green), and collagen structures (blue) fluorescence. White lines identify the magnified area in which Av.SRho fluorescence was modeled. Scale bars: $20 \mu \mathrm{m}$. Arrows indicate exteriorized Av.SRho+ granule structures. (B) Fold change in dextran-FITC mean fluorescence intensity (MFI) in the interstitial space following injection of DNP-HSA (green circles) or vehicle (open circles). The arrow indicates when the first exteriorized Av.SRho granule structures were detected. (C-F) Av.SRho (5 $\mu \mathrm{g}$, right ear, red circles or bars) or vehicle control (left ear, black or open circles or bars) was 
injected i.d. into the ear pinna of C57BL/6 mice. One week later mice were sensitized by i.d. injection of 20 ng of mouse anti-DNP IgE into both ear pinnae. Sixteen hours later, mice were injected i.p. with $500 \mu \mathrm{g}$ of DNP-HSA (Ag) or with vehicle (control) (open circles or bars). (C) Changes ( $\Delta$ ) in ear thickness over time after i.p. injection. (D) Representative H\&E (upper panel) and toluidine blue (TB, lower panel) photomicrographs of sections of ear pinnae in mice sacrificed 360 minutes after i.p. injection. Scale bars: $200 \mu \mathrm{m}$; asterisks indicate areas shown at higher magnification in the insets ( $\times 60)$. (E) Morphological assessment of MC degranulation in TB-stained sections 360 minutes after i.p. injection of Ag. Percentage and intensity of MC degranulation, as determined by classification into 3 categories: not degranulated (None [ $<10 \%$ of granules altered], open bars); moderately degranulated (Mod [10\%-50\% of granules altered], gray bars); and extensively degranulated (Ext [ $>50 \%$ of granules altered], black bars). (F) Number of leukocytes $/ \mathrm{mm}^{2}$ dermis 360 minutes after i.p. injection. (B and C) Mean \pm SEM; (E and F) mean + SEM; circles show values for individual mice. (B) Two-way ANOVA, (C, E, and F) 2-tailed, unpaired $t$ test (in $\mathbf{C}$, red and black asterisks represent significance of differences between data depicted by red or black circles vs. open circles, respectively). Data ( $n=3-12$ mice per group) are pooled from the 3 independent experiments performed (each done with $n=1-4$ mice per group), each of which gave similar results. ${ }^{*} P<0.05 ;{ }^{* *} P<0.01 ;{ }^{* *} P<0.001$. ns, not significant.

up to 6 hours after challenge in both Av.SRho ${ }^{+}$and Av.SRho ear pinnae (Figure 2C). Moreover, histological analyses revealed similar levels of MC degranulation and leukocyte infiltration in ear pinnae 6 hours after challenge regardless of the presence or absence of Av.SRho (Figure 2, D-F).

Taken together, these results indicate that the features of the IgE- and MC-dependent vascular responses and tissue inflammation we analyzed, which are thought to depend, at least in part, on the activities of mediators prestored in the cytoplasmic granules of MCs (29-32), were not substantially altered by the presence of fluorochrome-labeled avidin. These findings support the conclusion that i.d. injection of fluorochrome-labeled avidin not only represents an easy and fast way to label dermal MC secretory granules for their monitoring in vivo, but also will enable studies of MC degranulation in vivo in different disease models and, in principle, regardless of the mouse's genetic background.

Intravital monitoring of MC degranulation and activation of Illo gene transcription during CHS reactions. We next used our intravital imaging method to analyze, over time in the same animals, the spatiotemporal characteristics of MC degranulation and activation of $I l 10$ gene transcription in severe CHS reactions in situ (Supplemental Figure 2A). We first injected Av.SRho i.d. into the ear pinnae of Mcpt5EYFP mice (as described in Figure 1, A, E, and F) to monitor MC granule release over time at the site of CHS and to discriminate unambiguously Av.SRho ${ }^{+}$degranulated MCs from other cell types that potentially could have taken up free exteriorized Av.SRho ${ }^{+}$granules (e.g., dermal dendritic cells). Before DNFB challenge (day 0), only $\sim 10 \%$ of dermal MCs were marginally degranulated, exhibiting just a few Av.SRho ${ }^{+}$granules outside of the cell (Figure 3, A and C). However, as early as 1 day after DNFB challenge, $\sim 60 \%$ of dermal MCs were extensively degranulated and this number reached $\sim 90 \%$ at day 2 (Figure 3, A and C). Even though MCs were strongly degranulated at 2 and 3 days after DNFB challenge, most of the cells detected as strongly positive for Av.SRho in the analyzed areas were still identifiable as $\mathrm{EYFP}^{+}$dermal MCs (Figure 3A).

We then injected Av.SRho i.d. into ear pinnae of IL-10-GFP mice expressing a GFP tracker under the control of the $I l 10$ promoter (33), to monitor simultaneously over time both MC secretory granules and activation of $I l 10$ gene transcription. Before DNFB challenge (day 0), no Av.SRho ${ }^{+}$dermal MCs were positive for GFP, suggesting that, at least under these baseline conditions, the $I l 10$ gene was not substantially activated (Figure 3, B and C). However, we detected a clear GFP signal (i.e., emission of green fluorescence detectable above the green autofluorescence of the dermis) in $\sim 40 \%$ of Av.SRho ${ }^{+} \mathrm{MCs}$ as soon as 1 day after DNFB challenge, a percentage that remained stable for the next 2 days (Figure 3 , B and C). We next quantified the total number of IL-10-GFP+ cells at sites of CHS, and how many of these cells were Av.SRho ${ }^{+}$MCs. We observed a progressive increase over time in the total number of IL-10-GFP ${ }^{+}$cells (with the highest numbers 2 days after DNFB challenge) (Figure 3, B and D), a finding which is consistent with previous reports describing the kinetics of infiltration of Treg cells at sites of CHS (34). Notably, IL10-GFP ${ }^{+}$Av.SRho ${ }^{+}$dermal MCs represented up to $~ 55 \%$ of all detected IL-10-GFP ${ }^{+}$cells at day 1, but only $\sim 10 \%-20 \%$ at days 2 and 3 after DNFB challenge (Figure 3, B, D, and E). Taken together, these results suggest that dermal MCs are one of the first immune cells to produce IL-10 at sites of severe CHS, before the substantial infiltration of other IL-10-producing immune cells.

Evidence that MCs and MC-derived IL-10 limit features of severe CHS reactions. We next used genetic approaches to reassess the roles of MCs and MC-derived IL-10 in this model of severe CHS (Supplemental Figure 2A). In accord with our previous report (6), $\mathrm{Kit}^{W-s h / W-s h} \mathrm{MC}$-deficient mice exhibited greater ear swelling than their respective MC-sufficient littermate controls in this model (Supplemental Figure 2, B and C). Because $K i t^{W-s h / W-s h}$ mice are known to have many phenotypic abnormalities beside their MC 
A

Before DNFB challenge

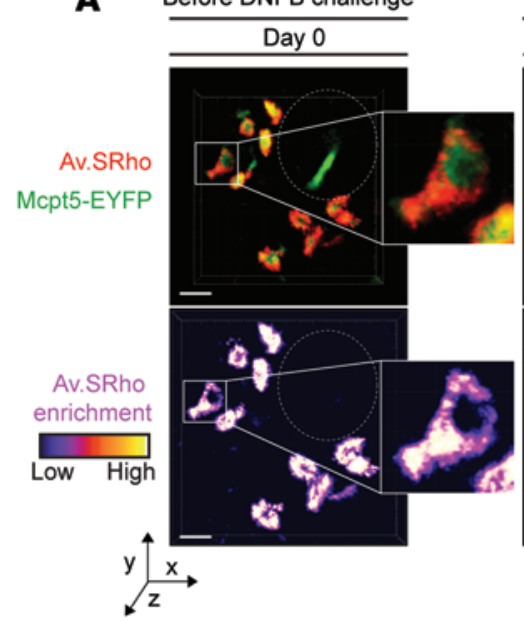

After DNFB challenge

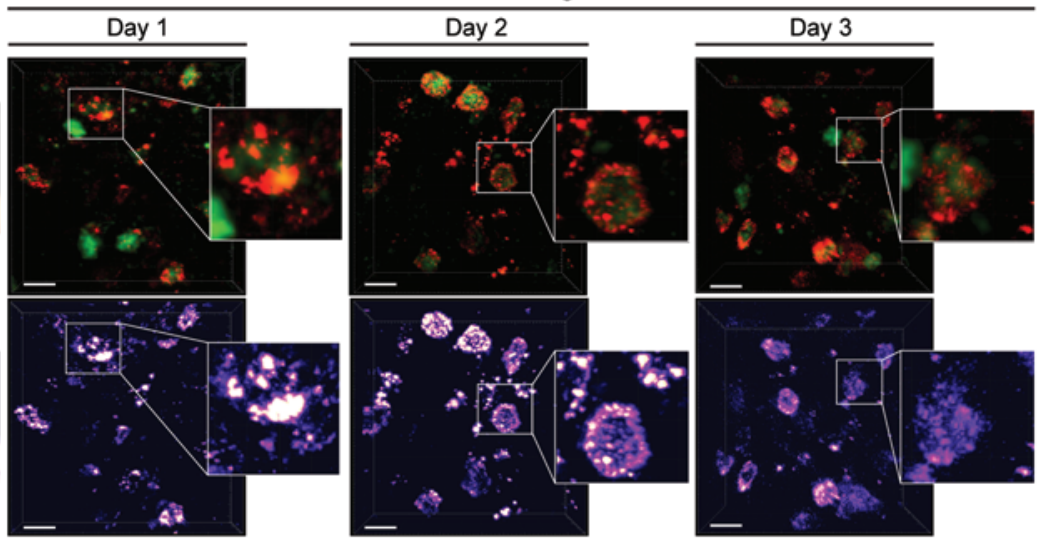

B

$\frac{\text { Before DNFB challenge }}{\text { Day } 0}$

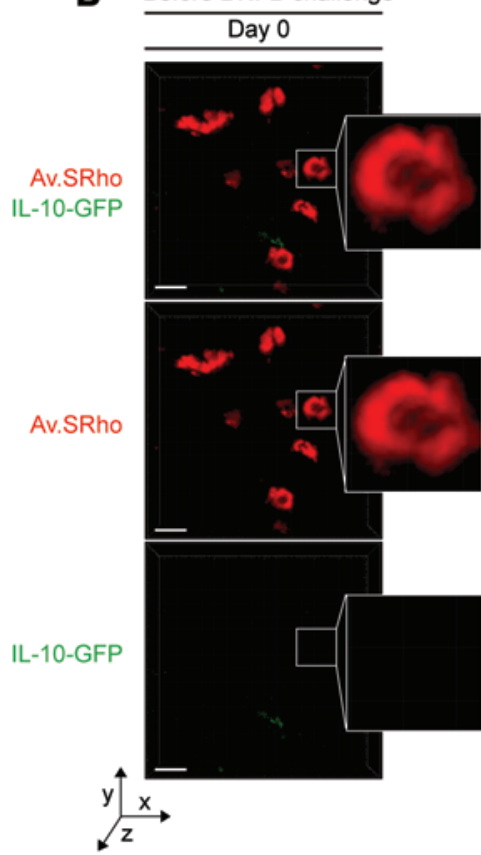

C - Mcpt5-EYFP+ ${ }^{+}$with exteriorized

Av.SRho+ structures in ear pinnae

- - Av.SRho+ IL-10-GFP' in ear pinnae

(mast cells expressing the il10 gene)

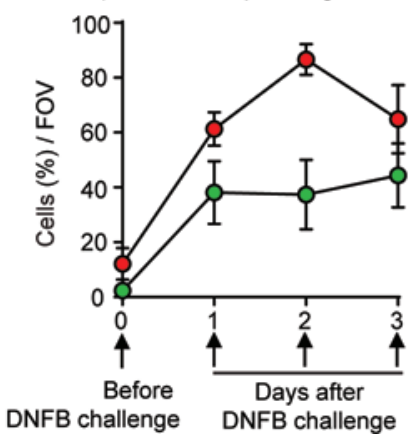

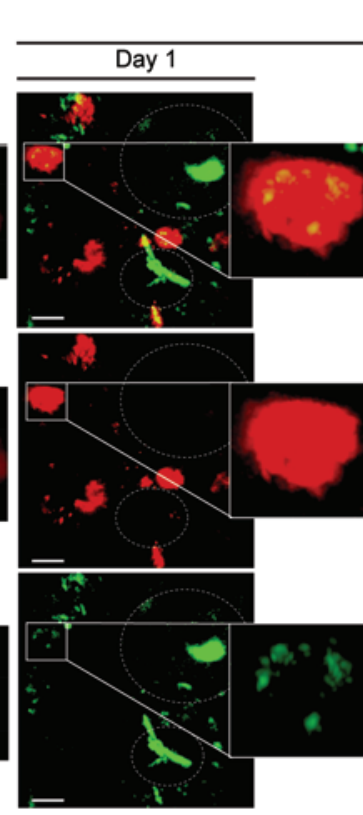

After DNFB challenge

\section{Day 2}
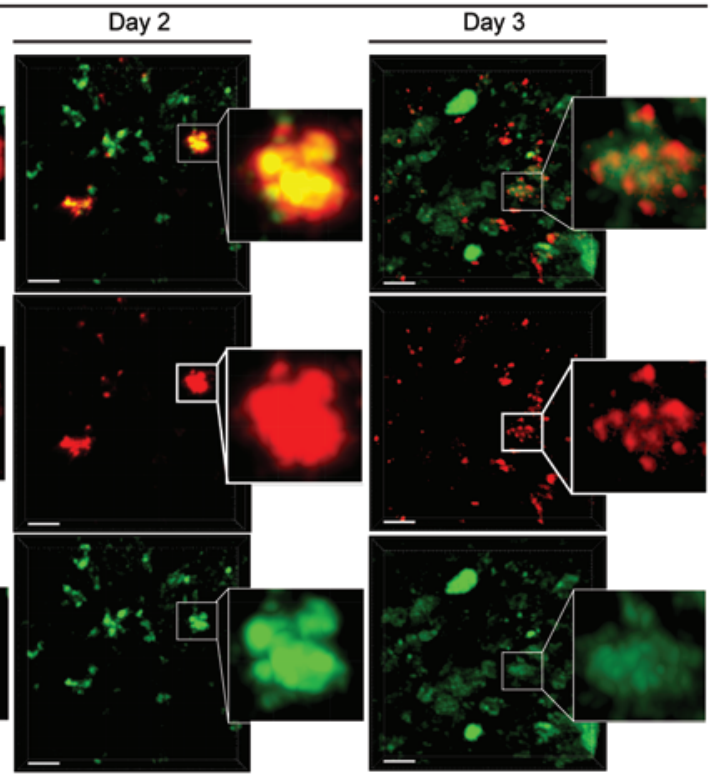

D
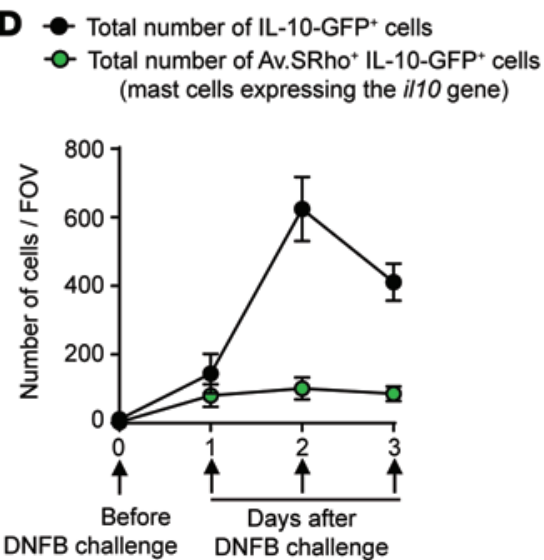

E Day 1 after DNFB challenge

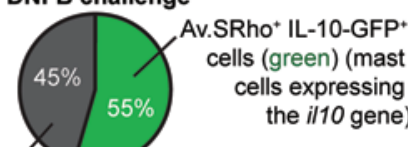

Av.SRho' IL-10-GFP+ cells (grey)

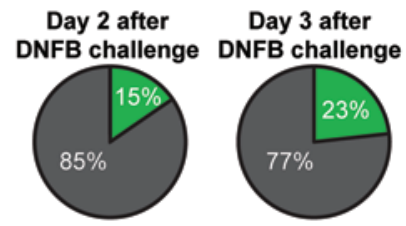

Figure 3. Longitudinal imaging of mast cell (MC) degranulation and II0 gene activation in a model of severe CHS. Sulforhodamine 101-coupled avidin (Av.SRho; $5 \mu \mathrm{g}$ ) was injected intradermally (i.d.) into the ear pinna of mice. One week later, the mice were treated as depicted in Supplemental Figure 2A to induce a severe 1-fluoro-2,4-dinitrobenzene-induced (DNFB-induced) contact hypersensitivity (CHS) reaction. (A) Longitudinal monitoring of the release of Av.SRho+ granules by dermal MCs at the site of CHS using intravital 2-photon microscopy. Representative 3D photographs of the ear pinna before DNFB challenge or at day 1, 2, or 3 after DNFB challenge. Upper panel: merged fluorescence of Av.SRho (red) and Mcpt5-EYFP (green). 
Lower panel: Av.SRho fluorescence (pseudocolor scale). Dashed white circles identify hair follicles. (B) Longitudinal monitoring of both the release of dermal MC Av.SRho+ granules and activation of $/ / 10$ gene transcription (IL-10-GFP, as detected by emission of GFP fluorescent signal) at the site of CHS using intravital 2-photon microscopy. Representative 3D photographs of the ear pinna before DNFB challenge or at day 1, 2, or 3 after DNFB challenge. Upper panel: merged fluorescence of Av.SRho (red) and IL-10-GFP (green). Middle panel: Av.SRho (red) fluorescence. Lower panel: IL-10-GFP (green) fluorescence. White lines identify the magnified areas and dashed white circles identify hair follicles. Scale bars: $20 \mu \mathrm{m}$. (C) Percentage of Mcpt5-EYFP+ cells with exteriorized Av.SRho+ structures (i.e., degranulated dermal MCs, red circles) and of Av.SRho+ IL-10-GFP+ cells (i.e., representing MCs expressing the $/ / 10$ gene, green circles) per field of view (FOV) in ear pinnae. (D) Total number of Av.SRho+ IL-10-GFP+ cells (MCs expressing the I/10 gene, green circles) per FOV in ear pinnae and total number of IL-10-GFP+ cells in ear pinnae (black circles). (E) Percentage of Av.SRho+ IL-10-GFP+ cells (i.e., representing MCs expressing the $/ / 10$ gene, green) and of Av.SRho-IL-10-GFP+ cells (i.e., representing other cell types expressing the $/ / 10$ gene, gray) among total IL-10-GFP+ cells in ear pinnae per FOV. Mean \pm SEM; data ( $n=3$ per group) are pooled from the 3 independent experiments performed (each done with 1 mouse per group), each of which gave similar results.

deficiency (10-12, 35-37), we also tested 2 other types of MC-deficient mice: $\mathrm{Cpa3}_{-\mathrm{Cre}}$; $\mathrm{Mcl}^{\mathrm{fl} / \mathrm{fl}}$ (9) and $M c p t 5-\mathrm{Cre}^{+}$; DTA mice (as used in ref. 7), both of which are Kit-independent MC-deficient mice.

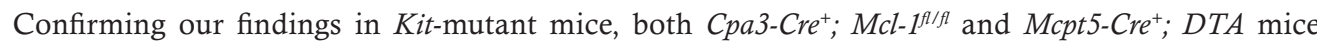
exhibited significantly enhanced ear swelling and epidermal hyperplasia compared with the values in their respective littermate controls (Figure 4 and Supplemental Figure 2, D and E). However, while $K i t^{W-s h / W-s h}$ mice exhibited an $\sim 200 \%$ increase in ear swelling on day 5 of the reaction as compared with their littermate controls (Supplemental Figure 2C), this difference was less pronounced in Kitindependent MC-deficient mice at the same time point ( $120 \%$ increase in $\mathrm{Cpa3}_{-} \mathrm{Cre}^{+}$; $\mathrm{Mcl}^{1 / 1 / f l}$ mice and $\sim 50 \%$ increase in $\mathrm{Mcpt}_{5}-\mathrm{Cre}^{+} ; \mathrm{DTA}$ mice) (Figure 4B and Supplemental Figure 2E). We think that the simplest interpretation of these results is that MCs can have effects that can significantly limit the assessed features of this acute model of severe CHS in each of the 3 examined mouse strains, but that additional phenotypic abnormalities in Kit ${ }^{W-s h / W-s h}$ mice (beside their MC deficiency) probably also contribute to the exacerbation of severe CHS responses in this strain. We then tested mice in which the Il10 gene was floxed out specifically in connective tissue-type MCs by generating $\mathrm{Mcpt5-Cre^{+ }}$; $I l 11^{q / f t}$ mice (Figure 5A). Dermal MCs were present in similar numbers in the ear pinnae of $\mathrm{Mcpt5}_{\mathrm{C}} \mathrm{Cr}^{+}$; $\mathrm{Il10^{2/fl }}$ mice (in which connective tissue-type MCs are deficient for IL-10) and littermate control Mcpt5-Cre- $\mathrm{Il1}^{\mathrm{q} / \mathrm{fl}}$

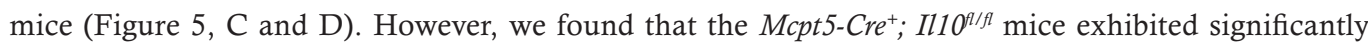
enhanced ear swelling and epidermal hyperplasia compared with the littermate control mice (Figure 5, B, C, and E). Notably, the enhancement of both the tissue swelling and the epidermal thickness associated with the reactions observed in $\mathrm{Mcpt5-Cre+}$; $1110^{a / f l}$ mice was less pronounced than that observed in the Kit-independent MC-deficient mice, suggesting that MCs might help to limit these features of this acute model of severe CHS by both IL-10-dependent and IL-10-independent mechanisms.

Taken together, these results indicate that MCs, and MC-derived IL-10, can help to limit the tissue changes associated with this model of severe CHS.

\section{Discussion}

In this study, we demonstrate the utility of a simple, fast, and inexpensive new in vivo imaging method that allows longitudinal intravital monitoring of the storage, release, and movement of MC secretory granules during immune responses, as exemplified here using both PCA and CHS models. Because this imaging method is based on the uptake of the fluorescent reporter, Av.SRho, by living dermal MCs and its long-term storage in their secretory granules, it can be used in mice of any genotype (as long as their MCs have secretory granules containing heparin or other highly negatively charged proteoglycans). This greatly increases the utility of this new imaging method compared with creating mouse strains in which fluorescent reporters are expressed in MCs based on genetic mechanisms.

By combining this new imaging method with genetic approaches, we provide additional evidence that MCs, and MC-derived IL-10, can help to limit the inflammation and other pathology at sites of severe hapten-induced CHS reactions. Notably, in a different disease setting, and using both $\mathrm{Kit}^{T-\mathrm{H} h / W \cdot \mathrm{sh}}$ mice and $\mathrm{Mcpt5}$-Cre $^{+}$; Il10//f mice, Chan et al. (38) provided evidence that MC production of IL-10 can suppress humoral and cell-mediated responses and enhance bacterial persistence in a mouse model of bladder infection with E. coli. The findings of Chan et al. (38) thus indicate that MC production of IL-10 also can contribute to suppression of inflammation in sites other than the skin.

However, we thought it was important to try to understand the possible reason(s) for the discrepancies between our results (obtained here and in our previous study; see ref. 6) and the results reported by 
A

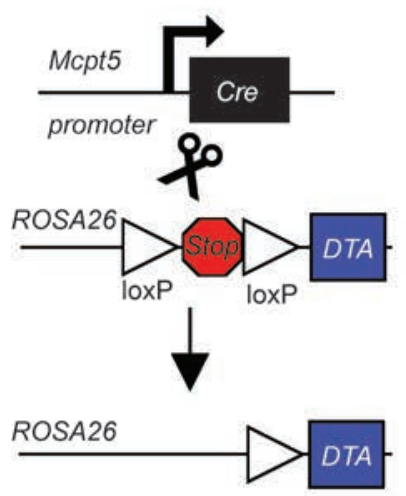

Mouse deficient for connective-tissue type mast cells

D

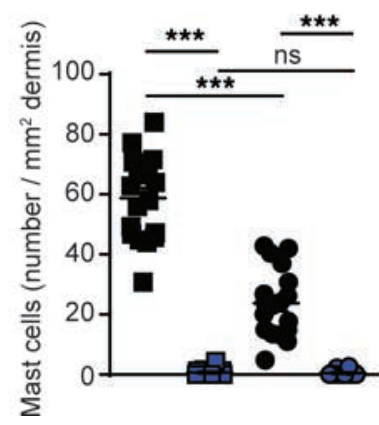

B
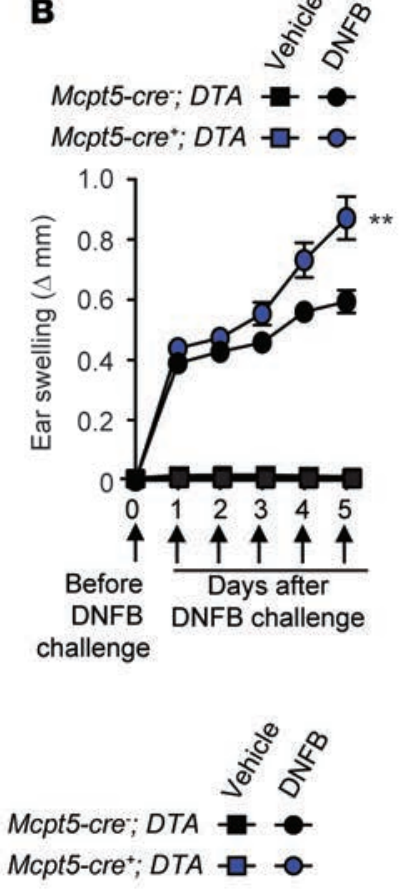

E

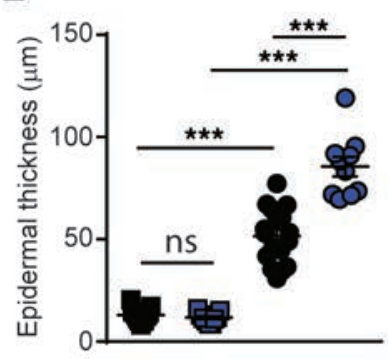

Day 5 after vehicle or DNFB challenge

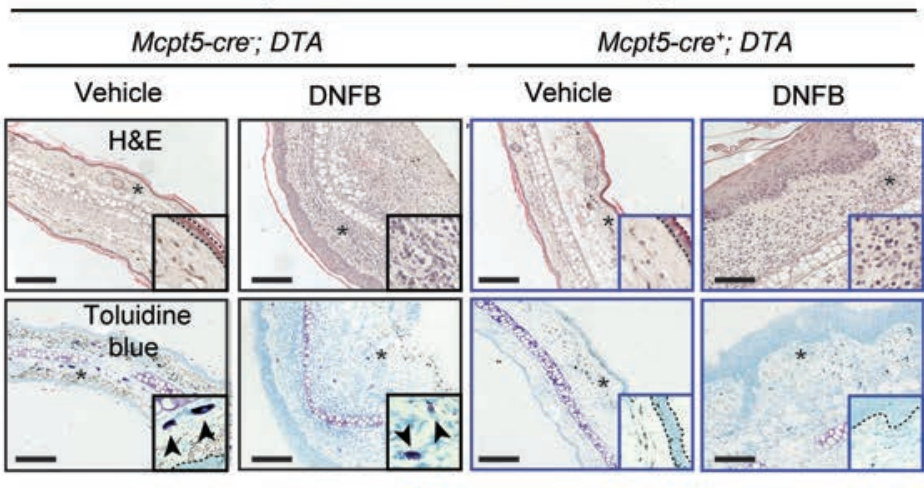

Figure 4. Connective tissue mast cells (MCs) limit inflammation and epidermal hyperplasia in a model of severe contact hypersensitivity (CHS). Mice were treated as depicted in Supplemental Figure 2A to elicit a 1-fluoro-2,4-dinitrobenzene-induced (DNFB-induced) severe CHS reaction. (A) Breeding strategy to obtain Mcpt5-Cre+; DTA+ (MC-deficient) mice. (B) Changes $(\Delta)$ in ear thickness over time after challenge with vehicle (squares) or DNFB (circles) in Mcpt5-Cre+; DTA+ (MC-deficient, blue) or Mcpt5-Cre-; DTA+ (MC-sufficient, black) mice. (C) Photomicrographs of representative H\&E (upper panel) and toluidine blue (lower panel) stained sections of ear pinnae of mice sacrificed 5 days after challenge. Asterisks indicate areas shown at higher magnification ( $\times 60)$ in insets, arrowheads indicate MCs, and dashed white lines in insets depict epidermis. (D) Number of MCs $/ \mathrm{mm}^{2}$ dermis and (E) epidermal thickness 5 days after vehicle (squares) or DNFB (circles) challenge in Mcpt5-Cre+; DTA+ (MC-deficient, blue) or Mcpt5-Cre'; DTA+ (MC-sufficient, black) mice. Scale bars: $200 \mu \mathrm{m}$. Mean $\pm \mathrm{SEM}$; ${ }^{* *} P<0.01$; ${ }^{* *} P<0.001$; (B) 2-way ANOVA; ( $\mathbf{D}$ and $\left.\mathbf{E}\right)$ 2-tailed, unpaired $t$ test. Data $(n=6-12$ mice per group) are pooled from the 3 independent experiments performed (each done with $n=2-4$ mice per group), each of which gave similar results. ns, not significant.

Dudeck et al. (7). We noticed that the features of the model of CHS we studied differed markedly from the one analyzed by Dudeck et al., e.g., the tissue swelling we observed in our model of severe CHS in WT mice (a stable increase in ear thickness of $\sim 0.2 \mathrm{~mm}$ on days $1-5$ of the reaction in Grimbaldeston et al. [see Figure 1B in ref. 6], and $\sim 0.25 \mathrm{~mm}$ in the present study [see Supplemental Figure 2C]) was much greater than that reported by Dudeck et al. ( $\sim 10 \%-25 \%$ increase in ear thickness at day 5 , diminished from values on days 1-2 [see Figure 2, A and F in ref. 7]). To confirm that MCs can indeed have proinflammatory effects in milder models of CHS (as has been proposed based on the findings of several prior studies employing Kit-mutant MC-deficient mice; see refs. 7, 39-41) and IgE-deficient mice (40), we tested mice using relatively low doses of DNFB for both the sensitization and the challenge phases, and we used a vehicle composed of a 4:1 mixture of acetone and olive oil for the challenge phase (as was employed by Dudeck et al. in ref. 7) (Supplemental Figure 3A). In contrast with the results obtained in our model of severe CHS (Supplemental Figure 2, D and E), we found that, in this model of mild CHS, both the ear swelling and the increase in epidermal thickness elicited were significantly lower in $\mathrm{Cpa3}-\mathrm{Cre} \mathrm{Fcl}^{+} \mathrm{Mcl}^{1 / 1 / \mathrm{f}}$ (Kit-independent) MC-deficient mice compared with the values in the corresponding MC-sufficient littermate control mice (Supplemental Figure 3, B and C). Finally, by intravital microscopy, we detected only minimal, if any, changes from baseline levels of MC Illo gene expression in this model of mild CHS (Supplemental Figure 4). Taken together, our results suggest that the extent of MC production of IL-10 during CHS responses may vary based on the severity of the model of CHS tested. 
A

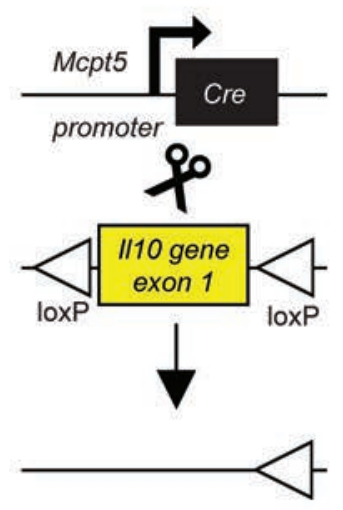

Skin and peritoneal mast cells deficient for il10
B

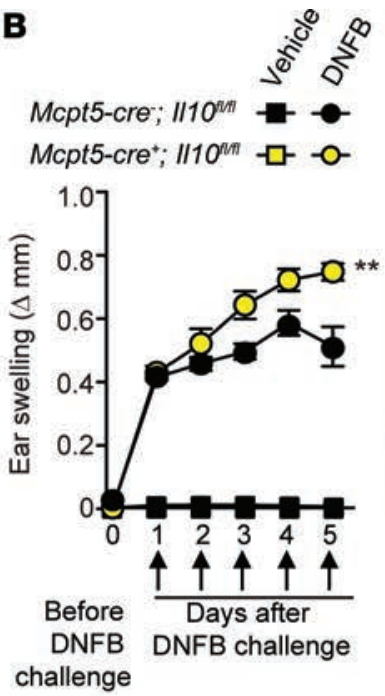

C

Day 5 after vehicle or DNFB challenge

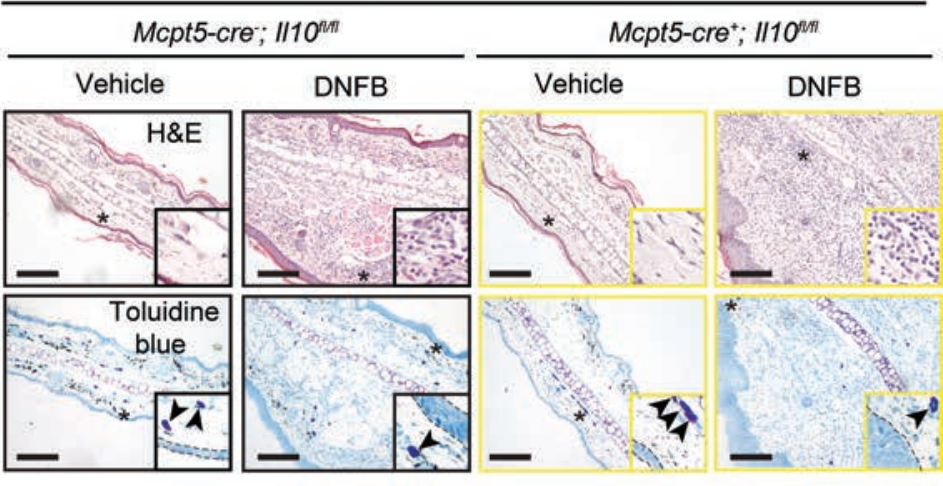

E

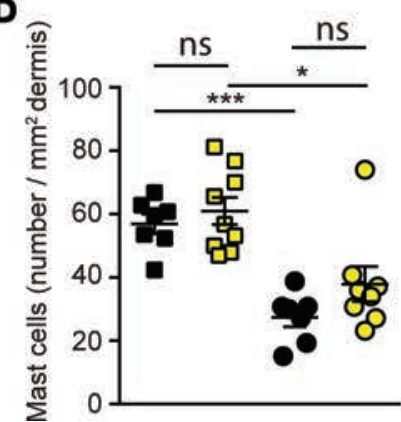

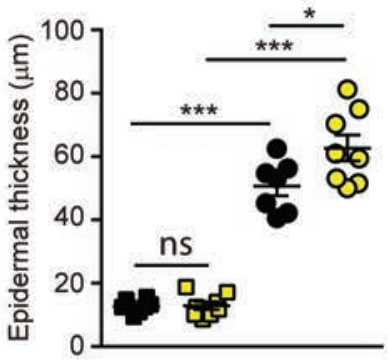

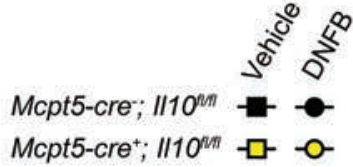

Mcpt5-cre+; II10"in - $\square-0$

Figure 5. Mast cell (MC) production of IL-10 limits inflammation and epidermal hyperplasia in a model of severe contact hypersensitivity (CHS). Mice were treated as depicted in Supplemental Figure 2A to elicit a 1-fluoro-2,4-dinitrobenzene-induced (DNFB-induced) severe CHS reaction. (A) Breeding strategy to obtain Mcpt5-Cre+ $/ 110^{f / f l}$ (MC IL-10 deficient) mice. (B) Changes $(\Delta)$ in ear thickness over time after challenge with vehicle

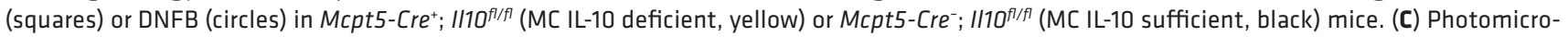
graphs of representative H\&E (upper panel) and toluidine blue (lower panel) stained sections of ear pinnae of mice sacrificed 5 days after challenge. Asterisks indicate areas shown at higher magnification $(\times 60)$ in insets, arrowheads indicate MCs, and dashed white lines in insets depict epidermis. (D) Number of MCs $/ \mathrm{mm}^{2}$ dermis and (E) epidermal thickness 5 days after vehicle (squares) or DNFB (circles) challenge in Mcpt5-Cre+; //10f/fl (MC IL-10 deficient, yellow) or Mcpt5-Cre-; /110 f/fl (MC IL-10 sufficient, black) mice. Scale bars: $200 \mu \mathrm{m}$. Mean $\pm \mathrm{SEM}$; ${ }^{*} P<0.05 ;{ }^{* *} P<0.01$; ${ }^{* * *} P<0.001$; (B) 2-way ANOVA; ( $\mathbf{D}$ and $\mathbf{E}$ ) 2-tailed, unpaired $t$ test. Data ( $n=6-12$ mice per group) are pooled from the 3 independent experiments performed (each done with $n=2-4$ mice per group), each of which gave similar results.

Taken together, our results help to reconcile the findings reported by Grimbaldeston et al. (6) and Dudeck et al. (7) by providing additional evidence that MCs can either amplify or limit the features of CHS reactions, depending on the severity of the model of CHS tested. Notably, Dudeck et al. (7) reported that, in models of CHS whose severity based on ear swelling measurements were intermediate between the severe and mild models of acute CHS used in the present study, MCs were important

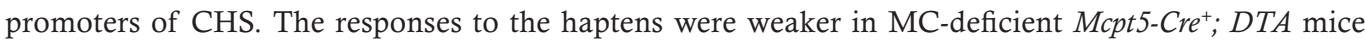
than in the corresponding MC-sufficient control mice, while the opposite result was obtained in MCdeficient $K i t^{W-s h / W-s h}$ mice. Also in the present study, we found that MCs can promote CHS of mild inten-

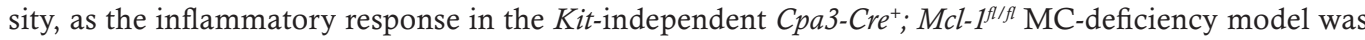
strongly reduced. It is possible that, for certain CHS reactions of moderate intensity, such proinflammatory MC functions may be concealed in Kit-mutant models by mechanisms of suppression of CHS that are KIT related but MC independent. Nevertheless, in contrast to the CHS-promoting MC functions observed in lower intensity CHS reactions, we report herein that in the severe model of DNFB-induced CHS used by Grimbaldeston et al. (6) a negative regulatory role for MCs and MC-derived IL-10 can be observed in mice that lack abnormalities of Kit structure or expression, including in the same mouse strains that were employed by Dudeck et al. in their report (7). 


\section{Methods}

\section{Study approval}

All animal care and experimentation was conducted in compliance with the guidelines of the NIH and with the specific approval of the Institutional Animal Care and Use Committees of Stanford University.

Mice

C57BL /6J (WT) mice were obtained from Jackson Laboratories and either bred at the Stanford University Research Animal Facility or used for experiments after maintaining the mice for at least 2 weeks in our animal facility. C57BL/6-Kit ${ }^{W-s h / W-s h}$ mice were originally provided by Peter Besmer (Molecular Biology Program, Memorial Sloan-Kettering Cancer Center, New York, NY, USA); we then backcrossed these mice to $C 57 B L / 6 J$ mice for more than 11 generations (42). Cpa3-Cre; $M c l-1^{f l f l}$ mice (backcrossed to $C 57 B L / 6$ for at least 9 generations) (9) were bred and maintained at the Stanford University Research Animal Facility. C57BL/6-Mcpt5-Cre+ mice were provided by Axel Roers (Institute for Immunology, University of Technology Dresden, Medical Faculty Carl-Gustav Carus, Dresden, Germany) and bred at the Stanford University Research Animal Facility. Il10 $0^{f / f l}$ mice (43) were kindly provided by Susan Kaech

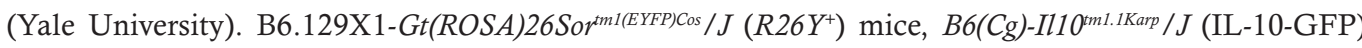

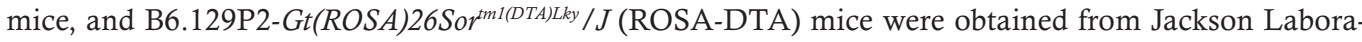
tories and bred at the Stanford University Research Animal Facility. We used age-matched mice of both sexes for the experiments, unless otherwise specified.

\section{Reagents}

DNP-HSA (catalog A6661), DNFB (catalog D1529), olive oil (catalog O1514), Av.SRho (catalog A2348), dextran-FITC (catalog 46945), Triton X-100 (catalog T8787), and poly-D-lysine (catalog P6407) were all from Sigma-Aldrich. DNP-specific IgE antibodies (clone $\varepsilon$-26) were provided by FuTong Liu (UC Davis, Davis, California, USA). Anti-KIT-Alexa Fluor 647 (A647) (clone 2B8, catalog 105818) was obtained from Biolegend. Nunc Lab-Tek 1.0 borosilicate cover glass system 8 chambers (catalog 155411) were from Thermo Fisher Scientific.

\section{CHS models}

Severe CHS model. We used the severe DNFB-induced model of CHS as described by Grimbaldeston et al. (6) and in Supplemental Figure 2A. Mice were sensitized on the shaved abdomen with $30 \mu 1$ of $0.5 \%$ (vol/ vol) DNFB in a vehicle of $100 \%$ acetone. At 5 days after sensitization, mice were challenged with $20 \mu 1$ of vehicle (100\% acetone) alone to 1 ear (10 $\mu 1$ to each side) and $0.2 \%$ (vol/vol) DNFB (in $100 \%$ acetone) to the other ear $(10 \mu$ to each side).

Mild CHS model. In the experiments described in Supplemental Figures 3 and 4, we used a more mild CHS model. Mice were sensitized on the shaved abdomen with $30 \mu 1$ of $0.2 \%$ (vol/vol) DNFB in a vehicle of $100 \%$ acetone. At 5 days after sensitization, mice were challenged with $20 \mu \mathrm{l}$ of vehicle (acetone/olive oil, $4: 1)$ alone to 1 ear (10 $\mu 1$ to each side) and $0.1 \%$ (vol/vol) DNFB (in acetone/olive oil, 4:1) to the other ear (10 $\mu 1$ to each side).

In both models of CHS, ear thickness was measured before and at several intervals after hapten challenge with a micrometer (Ozaki MFG). Toluidine blue and H\&E staining of histological sections of ear pinnae were performed as described in the supplemental methods section.

\section{Statistics}

Statistical tests were performed with PRISM 6 (GraphPad). Two-tailed Student's $t$ test (unpaired or paired) and 2-way ANOVA tests were performed as noted in the respective figure legends. ${ }^{*} P<0.05$; ${ }^{* *} P<0.01$; $* * * P<0.001 ; * * * * P<0.0001 ;$ ns, not significant $(P>0.05)$.

\section{Author contributions}

LLR, NG, and SJG conceived the project. LLR, RS, PS, MAG, MT, NG, and SJG were involved in experimental design. LLR and NG performed most of the experiments and compiled the data. RS and PS helped with experiments. AR provided mice. All authors participated in analyzing the data and writing or editing the paper. 


\section{Acknowledgments}

We thank all members of the Galli laboratory for discussions, Chen Liu and Mariola Liebersbach for technical assistance, and Susan Kaech (Yale University, New Haven, Connecticut, USA) for providing us with $I l 10^{q / f l}$ mice. L.L.R. acknowledges support from NIH grant K99AI110645, the European Commission (Marie Skłodowska-Curie Individual Fellowship H2020-MSCA-IF-2014 656086), and the Institut National de la Santé et de la Recherche Médicale (INSERM). R.S. was supported by a fellowship from the Lucile Packard Foundation for Children's Health and the Stanford NIH/NCRR CTSA award number UL1 RR025744. P.S. was supported by a Max Kade Fellowship of the Max Kade Foundation and the Austrian Academy of Sciences and a Schroedinger Fellowship of the Austrian Science Fund (FWF): J3399-B21. N.G. was supported by fellowships from the French "Fondation pour la Recherche Médicale FRM" award number SPE20130326582 and the Philippe Foundation. This work was supported by grants from the NIH (grants AI023990, CA072074, AI070813, AR067145 and U19AI104209 to S.J.G.), the United States-Israel Binational Science Foundation (grant 2013263 to S.J.G.), and the Department of Pathology, Stanford University.

Address correspondence to: Nicolas Gaudenzio or Stephen J. Galli, 269 Campus Drive, Room 3255b, Stanford, California 94305-5176, USA. Phone: 650.736.6014; E-mail: ngaudenz@stanford.edu (N. Gaudenzio); sgalli@stanford.edu (S.J. Galli).

1. Marshall JS. Mast-cell responses to pathogens. Nat Rev Immunol. 2004;4(10):787-799.

2. Theoharides TC, Kempuraj D, Tagen M, Conti P, Kalogeromitros D. Differential release of mast cell mediators and the pathogenesis of inflammation. Immunol Rev. 2007;217:65-78

3. Galli SJ, Grimbaldeston M, Tsai M. Immunomodulatory mast cells: negative, as well as positive, regulators of immunity. Nat Rev Immunol. 2008;8(6):478-486.

4. Abraham SN, St John AL. Mast cell-orchestrated immunity to pathogens. Nat Rev Immunol. 2010;10(6):440-452.

5. Galli SJ, Nakae S, Tsai M. Mast cells in the development of adaptive immune responses. Nat Immunol. 2005;6(2):135-142.

6. Grimbaldeston MA, Nakae S, Kalesnikoff J, Tsai M, Galli SJ. Mast cell-derived interleukin 10 limits skin pathology in contact dermatitis and chronic irradiation with ultraviolet B. Nat Immunol. 2007;8(10):1095-1104.

7. Dudeck A, et al. Mast cells are key promoters of contact allergy that mediate the adjuvant effects of haptens. Immunity. 2011;34(6):973-984.

8. Feyerabend TB, et al. Cre-mediated cell ablation contests mast cell contribution in models of antibody- and T cell-mediated autoimmunity. Immunity. 2011;35(5):832-844.

9. Lilla JN, et al. Reduced mast cell and basophil numbers and function in Cpa3-Cre; Mcl-1 ${ }^{\mathrm{f} / \mathrm{fl}}$ mice. Blood. 2011;118(26):6930-6938

10. Reber LL, Marichal T, Galli SJ. New models for analyzing mast cell functions in vivo. Trends Immunol. 2012;33(12):613-625.

11. Rodewald HR, Feyerabend TB. Widespread immunological functions of mast cells: fact or fiction? Immunity. 2012;37(1):13-24.

12. Galli SJ, Tsai M, Marichal T, Tchougounova E, Reber LL, Pejler G. Approaches for analyzing the roles of mast cells and their proteases in vivo. Adv Immunol. 2015;126:45-127.

13. Weber FC, et al. Neutrophils are required for both the sensitization and elicitation phase of contact hypersensitivity. $J$ Exp Med. 2015;212(1):15-22.

14. Gimenez-Rivera VA, Siebenhaar F, Zimmermann C, Siiskonen H, Metz M, Maurer M. Mast cells limit the exacerbation of chronic allergic contact dermatitis in response to repeated allergen exposure. J Immunol. 2016;197(11):4240-4246.

15. Gaudenzio N, et al. Different activation signals induce distinct mast cell degranulation strategies. J Clin Invest. 2016;126(10):3981-3998.

16. Dvorak AM. Human mast cells. Adv Anat Embryol Cell Biol. 1989;114:1-107.

17. Azouz NP, Hammel I, Sagi-Eisenberg R. Characterization of mast cell secretory granules and their cell biology. DNA Cell Biol. 2014;33(10):647-651.

18. Joulia R, et al. Mast cells form antibody-dependent degranulatory synapse for dedicated secretion and defence. Nat Commun. $2015 ; 6: 6174$.

19. Scholten J, et al. Mast cell-specific Cre/loxP-mediated recombination in vivo. Transgenic Res. 2008;17(2):307-315.

20. Egawa G, Nakamizo S, Natsuaki Y, Doi H, Miyachi Y, Kabashima K. Intravital analysis of vascular permeability in mice using two-photon microscopy. Sci Rep. 2013;3:1932.

21. Chen X, Nadiarynkh O, Plotnikov S, Campagnola PJ. Second harmonic generation microscopy for quantitative analysis of collagen fibrillar structure. Nat Protoc. 2012;7(4):654-669.

22. Zsebo KM, et al. Stem cell factor is encoded at the S1 locus of the mouse and is the ligand for the c-kit tyrosine kinase receptor. Cell. 1990;63(1):213-224.

23. Gaudenzio N, et al. Different activation signals induce distinct mast cell degranulation strategies. J Clin Invest. 2016;126(10):3981-3998.

24. Tharp MD, Seelig LL, Tigelaar RE, Bergstresser PR. Conjugated avidin binds to mast cell granules. J Histochem Cytochem. 1985;33(1):27-32

25. Wernersson S, Pejler G. Mast cell secretory granules: armed for battle. Nat Rev Immunol. 2014;14(7):478-494.

26. Kett WC, Osmond RI, Moe L, Skett SE, Kinnear BF, Coombe DR. Avidin is a heparin-binding protein. Affinity, specificity and structural analysis. Biochim Biophys Acta. 2003;1620(1-3):225-234.

27. Akahoshi M, et al. Mast cell chymase reduces the toxicity of Gila monster venom, scorpion venom, and vasoactive intestinal 
polypeptide in mice. J Clin Invest. 2011;121(10):4180-4191.

28. Schäfer B, et al. Mast cell anaphylatoxin receptor expression can enhance IgE-dependent skin inflammation in mice. $J$ Allergy Clin Immunol. 2013;131(2):541-548.e1.

29. Riley JF. Histamine in tissue mast cells. Science. 1953;118(3064):332.

30. Heltianu C, Simionescu M, Simionescu N. Histamine receptors of the microvascular endothelium revealed in situ with a histamine-ferritin conjugate: characteristic high-affinity binding sites in venules. J Cell Biol. 1982;93(2):357-364.

31. McLeod RL, Mingo GG, Kreutner W, Hey JA. Effect of combined histamine H1 and H3 receptor blockade on cutaneous microvascular permeability elicited by compound 48/80. Life Sci. 2005;76(16):1787-1794.

32. Kunder CA, St John AL, Abraham SN. Mast cell modulation of the vascular and lymphatic endothelium. Blood. 2011;118(20):5383-5393.

33. Madan R, et al. Nonredundant roles for B cell-derived IL-10 in immune counter-regulation. J Immunol. 2009;183(4):2312-2320.

34. Lehtimäki S, et al. The temporal and spatial dynamics of Foxp3+ Treg cell-mediated suppression during contact hypersensitivity responses in a murine model. J Invest Dermatol. 2012;132(12):2744-2751.

35. Gaudenzio N, Sibilano R, Stark1 P, Tsai M, Galli SJ, Reber LL. Analyzing the functions of mast cells in vivo using 'mast cell knock-in' mice. J Vis Exp. 2015;(99):e52753.

36. Gurish MF, Austen KF. Developmental origin and functional specialization of mast cell subsets. Immunity. 2012;37(1):25-33.

37. Grimbaldeston MA, Chen CC, Piliponsky AM, Tsai M, Tam SY, Galli SJ. Mast cell-deficient W-sash c-kit mutant Kit W-sh/W-sh mice as a model for investigating mast cell biology in vivo. Am J Pathol. 2005;167(3):835-848

38. Chan CY, St John AL, Abraham SN. Mast cell interleukin-10 drives localized tolerance in chronic bladder infection. Immunity. 2013;38(2):349-359

39. Biedermann T, et al. Mast cells control neutrophil recruitment during T cell-mediated delayed-type hypersensitivity reactions through tumor necrosis factor and macrophage inflammatory protein 2. J Exp Med. 2000;192(10):1441-1452.

40. Bryce PJ, Miller ML, Miyajima I, Tsai M, Galli SJ, Oettgen HC. Immune sensitization in the skin is enhanced by antigen-independent effects of IgE. Immunity. 2004;20(4):381-392.

41. Norman MU, et al. Mast cells regulate the magnitude and the cytokine microenvironment of the contact hypersensitivity response. Am J Pathol. 2008;172(6):1638-1649.

42. Piliponsky AM, et al. Mast cell-derived TNF can exacerbate mortality during severe bacterial infections in C57BL/6-KitWsh/W-sh mice. Am J Pathol. 2010;176(2):926-938

43. Roers A, et al. T cell-specific inactivation of the interleukin 10 gene in mice results in enhanced $\mathrm{T}$ cell responses but normal innate responses to lipopolysaccharide or skin irritation. J Exp Med. 2004;200(10):1289-1297. 